ANNALES

POLONICI MATHEMATICI

$86.1(2005)$

\title{
Univalence, strong starlikeness and integral transforms
}

\author{
by M. Obradović (Belgrad), S. Ponnusamy (Chennai), \\ and P. VAsundhra (Chennai)
}

\begin{abstract}
Let $\mathcal{A}$ represent the class of all normalized analytic functions $f$ in the unit disc $\Delta$. In the present work, we first obtain a necessary condition for convex functions in $\Delta$. Conditions are established for a certain combination of functions to be starlike or convex in $\Delta$. Also, using the Hadamard product as a tool, we obtain sufficient conditions for functions to be in the class of functions whose real part is positive. Moreover, we derive conditions on $f$ and $\mu$ so that the non-linear integral transform $\int_{0}^{z}(\zeta / f(\zeta))^{\mu} d \zeta$ is univalent in $\Delta$. Finally, we give sufficient conditions for functions to be strongly starlike of order $\alpha$.
\end{abstract}

1. Introduction. Let $\mathcal{H}$ denote the class of all functions analytic in the unit disc $\Delta=\{z:|z|<1\}$, and $\mathcal{A}$ the class of all normalized functions $f$ $\left(f(0)=f^{\prime}(0)-1=0\right)$ in $\mathcal{H}$. Let $\mathcal{S}$ denote the univalent subclass of $\mathcal{A}$, and $\mathcal{S}^{*}$ denote the subclass of $f \in \mathcal{S}$ for which $f(\Delta)$ is starlike with respect to the origin. Recall the prominent subclasses studied in the theory of univalent functions (see [7]), for $0 \leq \beta<1$ :

$$
\begin{aligned}
\mathcal{P}(\beta) & =\left\{f \in \mathcal{A}: \operatorname{Re}\left(\frac{f(z)}{z}\right)>\beta, z \in \Delta\right\}, \\
\mathcal{R}(\beta) & =\left\{f \in \mathcal{A}: z f^{\prime} \in \mathcal{P}(\beta)\right\}, \\
\mathcal{S}^{*}(\beta) & =\left\{f \in \mathcal{A}: \operatorname{Re}\left(\frac{z f^{\prime}(z)}{f(z)}\right)>\beta, z \in \Delta\right\}, \\
\mathcal{S}_{\beta}^{*} & =\left\{f \in \mathcal{A}:\left|\arg \left(\frac{z f^{\prime}(z)}{f(z)}\right)\right|<\frac{\beta \pi}{2}, z \in \Delta\right\}, \\
\mathcal{K}(\beta) & =\left\{f \in \mathcal{A}: z f^{\prime} \in \mathcal{S}^{*}(\beta)\right\} .
\end{aligned}
$$

It is well known that $\mathcal{K} \equiv \mathcal{K}(0) \subsetneq \mathcal{S}^{*}(1 / 2)$. Functions in $\mathcal{S}_{\beta}^{*}$ are called strongly starlike of order $\beta$, while those in $\mathcal{S}^{*}(\beta)$ are starlike of order $\beta$. For $\beta<0, \mathcal{S}^{*}(\beta) \nsubseteq \mathcal{S}$, while for $0<\beta<1, \mathcal{S}^{*}(\beta) \subsetneq \mathcal{S}^{*} \subsetneq \mathcal{S}$, and functions

2000 Mathematics Subject Classification: 30C45, 30C55.

Key words and phrases: univalent, starlike, strongly starlike and convex functions.

The authors thank the referee for his/her valuable comments. 
in $\mathcal{S}^{*}(0) \equiv \mathcal{S}^{*}$ are simply referred to as starlike. For $0<\beta<1$, clearly, $\mathcal{S}_{\beta}^{*} \subsetneq \mathcal{S}^{*}$ and $\mathcal{S}_{1}^{*} \equiv \mathcal{S}^{*}$.

For $a, b, c \in \mathbb{C}$ and $c \neq 0,-1,-2, \ldots$, the Gaussian hypergeometric series $F(a, b ; c ; z)$ is defined as

$$
F(a, b ; c ; z)=\sum_{n=0}^{\infty} \frac{(a)_{n}(b)_{n}}{(c)_{n}} \frac{z^{n}}{n !}, \quad|z|<1,
$$

where $(a)_{n}=a(a+1)(a+2) \cdots(a+n-1)$ and $(a)_{0}=1$. This series represents an analytic function in $\Delta$ and has an analytic continuation throughout the finite complex plane except at most for the cut $[1, \infty)$.

Let $\mathcal{B}$ denote another important subclass, of all analytic functions $\omega \in \mathcal{H}$ such that $\omega(0)=0$ and $\omega(\Delta) \subseteq \Delta$. A function $f \in \mathcal{H}$ is called subordinate to another function $g \in \mathcal{H}$, and one writes $f(z) \prec g(z)$, if there exists an $\omega \in \mathcal{B}$ such that $f(z)=g(\omega(z))$ for all $z \in \Delta$. It is well known that this implies in particular $f(0)=g(0)$ and $f(\Delta) \subset g(\Delta)$, and that these two conditions are also sufficient for $f(z) \prec g(z)$ whenever $g$ is univalent in $\Delta$. Next, we remark that if $f \in \mathcal{H}, f(0)=0$ and $|f(z)| \leq M$ on $\Delta$, then this can be equivalently expressed in the form

$$
f(z)=M \omega(z), \quad \omega \in \mathcal{B},
$$

and so $f(z) \prec M z$.

In [8], R. Singh and S. Paul showed that for all real $\lambda$ and $\mu$ with $0 \leq$ $\mu \leq \lambda / 2$ one has the following implication:

$$
f \in \mathcal{K} \Rightarrow \operatorname{Re}\left(\lambda \frac{f(z)}{z f^{\prime}(z)}+\mu \frac{1}{f^{\prime}(z)}\right)>0, \quad z \in \Delta .
$$

We observe that the well known strict inclusion result, namely $\mathcal{K} \subsetneq \mathcal{S}^{*}(1 / 2)$, does not follow from the above one way implication. In view of this, in Theorem 2.1 we use a different approach and determine $R=R(\lambda, \mu)$ such that

$$
f \in \mathcal{K} \Rightarrow G(\Delta) \subset\{w \in \mathbb{C}:|w-R|<R\}, \quad G(z)=\lambda \frac{f(z)}{z f^{\prime}(z)}+\mu \frac{1}{f^{\prime}(z)},
$$

for all real values of $\lambda$ and $\mu$ with $|\mu| \leq \lambda / 2$.

Trimble [11] showed that if $f \in \mathcal{K}$, then $F$ defined by

$$
F(z)=\lambda z+(1-\lambda) f(z)
$$

is in $\mathcal{S}^{*}(\beta)$, where $\beta=(1-3 \lambda) /(2(2+\lambda))$ with $0 \leq \lambda \leq 1 / 3$. Related problems were considered in $[2,12]$, by imposing an additional condition on $f$.

In Theorem 2.3, we impose conditions on $f \in \mathcal{A}_{n}:=\{f \in \mathcal{A}: f(z)=$ $\left.z+\sum_{k=n+1}^{\infty} a_{k} z^{k}\right\}$ different from those of $[2,12]$ and obtain the starlikeness 
of

$$
F(z)=\lambda z+\frac{1-\lambda}{\alpha} \int_{0}^{1} t^{1 / \alpha-2} f(t z) d t
$$

for all $\lambda<1$. It follows that the integral (1.2) is well defined or convergent only for $\operatorname{Re} \alpha>0$ and also at $\alpha=0$ as a limiting case, because

$$
\begin{aligned}
\frac{1}{\alpha} \int_{0}^{1} t^{1 / \alpha-2+k} d t & =\frac{1}{(k-1) \alpha+1}\left[1-\lim _{t \rightarrow 0^{+}} \exp \left(\left(\frac{1}{\alpha}-1+k\right) \ln t\right)\right] \\
& =\frac{1}{(k-1) \alpha+1},
\end{aligned}
$$

for $k=1, n+1, n+2, \ldots$, where the principal branches of possible multiplevalued power functions are considered. We remark that the relation (1.2) looks much simpler in the following differential form:

$$
\alpha z F^{\prime}(z)+(1-\alpha) F(z)=\lambda z+(1-\lambda) f(z)
$$

since

$$
f(z) \equiv \int_{0}^{1} \frac{\partial}{\partial t}\left(t^{1 / \alpha-1} f(t z)\right) d t .
$$

Thus, for a given $f \in \mathcal{A}_{n}$, there is exactly one solution $F \in \mathcal{A}_{n}$ of the equation (1.3) if and only if $\alpha \in \mathbb{C} \backslash\{-1 / j: j=n, n+1, n+2, \ldots\}$ :

$$
F(z) \equiv z+(1-\lambda) \sum_{k=n+1}^{\infty} \frac{a_{k}}{(k-1) \alpha+1} z^{k}
$$

whenever $f(z)=z+\sum_{k=n+1}^{\infty} a_{k} z^{k}$. We use this observation in the proof of Theorem 2.3.

Also, we provide a condition on $\beta$ such that $\operatorname{Re} z f^{\prime \prime}(z)>-\beta(1-\lambda)$ implies that $\operatorname{Re}(f(z) / z)>\lambda$ (see Theorem 2.6). In addition to these results, in Theorem 2.7, we obtain conditions so that the non-linear operator

$$
g(z)=\int_{0}^{z}\left(\frac{\zeta}{f(\zeta)}\right)^{\mu} d \zeta
$$

is univalent. Finally, we derive a sufficient condition for $f$ to be strongly starlike of order $\alpha$.

\section{Main results}

Theorem 2.1. If $f \in \mathcal{K}$ then

$$
\left|\lambda \frac{f(z)}{z f^{\prime}(z)}+\mu \frac{1}{f^{\prime}(z)}-\frac{\lambda(\lambda+2 \mu)}{\lambda-2 \mu}\right|<\frac{\lambda(\lambda+2 \mu)}{\lambda-2 \mu}, \quad z \in \Delta,
$$


for all real $\lambda$ and $\mu$ with $0<\mu \leq \lambda / 2$, and

$$
\left|\lambda \frac{f(z)}{z f^{\prime}(z)}+\mu \frac{1}{f^{\prime}(z)}-\lambda\right|<\lambda, \quad z \in \Delta,
$$

for all real $\lambda$ and $\mu$ with $-\lambda / 2 \leq \mu<0$.

Proof. Let $f \in \mathcal{K}$. Since $\mathcal{K} \subsetneq \mathcal{S}^{*}(1 / 2)$, we exclude the trivial case $\mu=$ $0<|\lambda|$ as this may be obtained as a limiting case. Then, for all $z$ and $w$ in $\Delta$, it is known that

$$
\operatorname{Re}\left(\frac{z f^{\prime}(z)}{f(z)-f(w)}-\frac{w}{z-w}\right)>\frac{1}{2}
$$

where the expression is defined by its limit when $z=w$. Further, for $f \in \mathcal{K}$ it is also known that $\operatorname{Re}(f(z) / z)>1 / 2$ in $\Delta$ and hence, for $0<\mu \leq \lambda / 2$, this shows that

$$
0<\operatorname{Re}\left(\frac{\mu z}{\mu z+\lambda f(z)}\right) \leq \frac{2 \mu}{\lambda+2 \mu} .
$$

Since $f \in \mathcal{K}$, the image of $f$ covers the disc $|\zeta|<1 / 2$ and therefore, it can be readily seen that there exists $w \in \Delta$ such that

$$
f(w)=-(\mu / \lambda) z
$$

From (2.3) and (2.4),

$$
\begin{aligned}
\operatorname{Re}\left(\frac{\lambda z f^{\prime}(z)}{\lambda f(z)+\mu z}\right) & =\operatorname{Re}\left(\frac{z f^{\prime}(z)}{f(z)-f(w)}\right) \\
& >\frac{1}{2}+\operatorname{Re}\left(\frac{w}{z-w}\right)=\frac{1}{2}-\operatorname{Re}\left(\frac{\mu w}{\mu w+\lambda f(w)}\right) \\
& >\frac{1}{2}-\frac{2 \mu}{\lambda+2 \mu}=\frac{\lambda-2 \mu}{2(\lambda+2 \mu)},
\end{aligned}
$$

which proves the first assertion (2.1) for $0<\mu<\lambda / 2$. If $\mu=\lambda / 2$, then the last inequality becomes

$$
\operatorname{Re}\left(\lambda \frac{f(z)}{z f^{\prime}(z)}+\frac{1}{2} \frac{1}{f^{\prime}(z)}\right)>0,
$$

which is same as (2.1) in the limiting case.

Next, we observe that for $-\lambda / 2 \leq \mu<0$,

$$
\operatorname{Re}\left(1+\frac{\lambda f(z)}{\mu z}\right)<\frac{\lambda+2 \mu}{2 \mu} \leq 0
$$

so that

$$
\frac{2 \mu}{\lambda+2 \mu}<\frac{1}{\operatorname{Re}(1+\lambda f(z) / \mu z)} \leq \operatorname{Re}\left(\frac{1}{1+\lambda f(z) / \mu z}\right)<0 .
$$


This observation shows that

$$
\operatorname{Re}\left(\frac{\lambda z f^{\prime}(z)}{\lambda f(z)+\mu z}\right)>\frac{1}{2}, \quad z \in \Delta,
$$

which proves the second assertion (2.2).

Corollary 2.2. Let $f \in \mathcal{K}$. For $z, w \in \Delta$, define

$$
G(z, w)=\lambda \frac{[f(z)-f(w)]\left(1-|w|^{2}\right)}{(z-w) f^{\prime}(z)(1-\bar{w} z)}+\mu \frac{f^{\prime}(w)\left(1-|w|^{2}\right)^{2}}{f^{\prime}(z)(1-\bar{w} z)^{2}} .
$$

Then, for all real $\lambda$ and $\mu$ such that $0<\mu \leq \lambda / 2$, we have

$$
\left|G(z, w)-\frac{\lambda(\lambda+2 \mu)}{\lambda-2 \mu}\right|<\frac{\lambda(\lambda+2 \mu)}{\lambda-2 \mu},
$$

and for $-\lambda / 2 \leq \mu<0$, we have $|G(z, w)-\lambda|<\lambda$.

Proof. Since $f^{\prime}(w) \neq 0$ in $\Delta$, we consider a disc automorphism of $\Delta$ and define $g$ by

$$
g(\zeta)=\frac{f((\zeta+w) /(1+\bar{w} \zeta))-f(w)}{f^{\prime}(w)\left(1-|w|^{2}\right)}
$$

As the convexity is preserved under disc automorphisms, we have $g \in \mathcal{K}$ if and only if $f \in \mathcal{K}$. Writing $z=(w+\zeta) /(1+\zeta \bar{w})$, it can be shown that

$$
\frac{\lambda g(\zeta)+\mu \zeta}{\zeta g^{\prime}(\zeta)}=G(z, w)
$$

where $G(z, w)$ is given by $(2.5)$. Since $g \in \mathcal{K}$, the desired conclusion follows from Theorem 2.1 and the last equality.

Theorem 2.3. Let $n \in \mathbb{N}, \alpha \in \mathbb{C} \backslash\{-1 / j: j=n, n+1, n+2, \ldots\}$ with $\operatorname{Re} \alpha>-1 / n$ and let $f \in \mathcal{A}_{n}$ satisfy the condition

$$
\left|z f^{\prime \prime}(z)\right|<\frac{\mu}{1-\lambda}, \quad z \in \Delta,
$$

for some $\lambda<1$. Then, for $F$ defined by (1.3), we have

(a) $\left|\frac{z F^{\prime}(z)}{F(z)}-1\right| \leq 1$ for $0<\mu \leq n \operatorname{Re} \alpha+1$,

(b) $\left|\frac{z F^{\prime \prime}(z)}{F^{\prime}(z)}\right| \leq 1$ for $0<\mu \leq(n \operatorname{Re} \alpha+1) / 2$.

Proof. From the representation (1.4), we easily see that

$$
z F^{\prime \prime}(z)=(1-\lambda) \sum_{k=n}^{\infty} \frac{(k+1) k a_{k+1} z^{k}}{1+k \alpha}=(1-\lambda)\left[z f^{\prime \prime}(z) *\left(\sum_{k=n}^{\infty} \frac{z^{k}}{1+k \alpha}\right)\right]
$$


and thus,

$$
z F^{\prime \prime}(z)=(1-\lambda) \int_{0}^{1} t^{\alpha} z f^{\prime \prime}\left(t^{\alpha} z\right) d t
$$

Suppose that $f$ satisfies condition (2.6), which may be rewritten as

$$
z f^{\prime \prime}(z)=\frac{\mu}{1-\lambda} \omega(z), \quad \omega \in \mathcal{B}_{n},
$$

where $\mathcal{B}_{n}=\left\{\omega \in \mathcal{H}:|\omega(z)|<1\right.$ and $\omega^{(k)}(0)=0$ for $\left.k=0,1, \ldots, n-1\right\}$. Schwarz' lemma then shows that $|\omega(z)| \leq|z|^{n}$ for $z \in \Delta$. Therefore, (2.7) becomes

$$
z F^{\prime \prime}(z)=\mu \int_{0}^{1} \omega\left(t^{\alpha} z\right) d t
$$

and hence, by the condition on $\alpha$, it follows that

$$
\left|z F^{\prime \prime}(z)\right| \leq \frac{\mu|z|^{n}}{n \operatorname{Re} \alpha+1}<\frac{\mu}{n \operatorname{Re} \alpha+1}, \quad z \in \Delta
$$

Then (see $[7,10])$ we have

$$
\left|\frac{z F^{\prime}(z)}{F(z)}-1\right| \leq \frac{\mu /[2 n \operatorname{Re} \alpha+2]}{1-\mu /[2 n \operatorname{Re} \alpha+2]}
$$

and

$$
\left|\frac{z F^{\prime \prime}(z)}{F^{\prime}(z)}\right| \leq \frac{\mu /[n \operatorname{Re} \alpha+1]}{1-\mu /[n \operatorname{Re} \alpha+1]} .
$$

In particular, $F$ is starlike for $0<\mu \leq n \operatorname{Re} \alpha+1$ and convex if $0<\mu \leq$ $(n \operatorname{Re} \alpha+1) / 2$.

The case $n=1$ of Theorem 2.3 gives

Corollary 2.4. Let $\operatorname{Re} \alpha>-1$ and let $f \in \mathcal{A}$ satisfy the condition

$$
\left|z f^{\prime \prime}(z)\right|<\frac{\mu}{1-\lambda}, \quad z \in \Delta
$$

for some $\lambda<1$. Then, for $F$ defined by (1.2), we have
(a) $\left|\frac{z F^{\prime}(z)}{F(z)}-1\right| \leq 1$ for $0<\mu \leq \operatorname{Re} \alpha+1$,
(b) $\left|\frac{z F^{\prime \prime}(z)}{F^{\prime}(z)}\right| \leq 1$ for $0<\mu \leq(\operatorname{Re} \alpha+1) / 2$.

Note that $z+(c / 2) z^{2} \notin \mathcal{S}$ whenever $|c|>1$. Define

$$
f(z)=z+(\mu / 2(1-\lambda)) z^{2} .
$$

Now, if we let $1-\mu<\lambda \leq 1$, then $\mu /(1-\lambda)>1$ and hence $f$ is not univalent but satisfies (2.10). On the other hand, the corresponding $F$ defined by (1.2) is starlike for $0<\mu \leq \operatorname{Re} \alpha+1$ and is in fact convex for $0<\mu \leq(\operatorname{Re} \alpha+1) / 2$. 
Lemma 2.5. Let $p$ be analytic in $\Delta$ and $p(0)=1$. Suppose that

$$
\operatorname{Re}\left(z^{2} p^{\prime \prime}(z)+\alpha z p^{\prime}(z)\right)>-\beta(1-\lambda), \quad z \in \Delta,
$$

for some $\alpha>1, \lambda<1$ and $0<\beta \leq \beta(\alpha)$, where

$$
\beta(\alpha):=\frac{\alpha(\alpha-1)}{2[\alpha \log 2-F(1, \alpha ; \alpha+1 ;-1)]} .
$$

Then $\operatorname{Re} p(z)>\lambda$ for $z \in \Delta$. In particular, if

$$
\operatorname{Re}\left(z^{2} p^{\prime \prime}(z)+\alpha z p^{\prime}(z)\right)>-\beta
$$

for $0<\beta \leq \beta(\alpha)$, then $\operatorname{Re} p(z)>0$ for $z \in \Delta$.

Proof. We consider a more general differential equation

$$
z^{2} p^{\prime \prime}(z)+\alpha z p^{\prime}(z)=\beta(1-\lambda)(\phi(z)-1)
$$

where $\operatorname{Re} \phi(z)>0$ in $\Delta$, and $\phi(0)=1$. If $p$ and $\phi$ are of the form

$$
p(z)=1+\sum_{n=1}^{\infty} p_{n} z^{n} \quad \text { and } \quad \phi(z)=1+\sum_{n=1}^{\infty} \phi_{n} z^{n},
$$

respectively, then, by comparing the coefficients of $z^{n}$ on both sides of (2.11), it follows that

$$
n(n-1+\alpha) p_{n}=\beta(1-\lambda) \phi_{n}, \quad n \geq 1,
$$

which gives

$$
p(z)=1+\beta(1-\lambda) \sum_{n=1}^{\infty} \frac{\phi_{n}}{n(n-1+\alpha)} z^{n} .
$$

It can be easily seen that $p(z)$ has the integral representation (see [5, Proposition 1])

$$
p(z)=1+\beta(1-\lambda) \int_{0}^{1} \int_{0}^{1} u^{-1} v^{\alpha-2}(\phi(u v z)-1) d u d v .
$$

As $\operatorname{Re} \phi(z)>(1-|z|) /(1+|z|)$ for $z \in \Delta$, we have

$$
\operatorname{Re}(\phi(u v z)-1) \geq-\frac{2|u v z|}{1+u v|z|} \geq-\frac{2 u v}{1+u v}, \quad z \in \Delta,
$$

and therefore,

$$
\begin{aligned}
\operatorname{Re} p(z) & >1-2 \beta(1-\lambda) \int_{0}^{1} \int_{0}^{1} \frac{v^{\alpha-1}}{1+u v} d u d v \\
& =1-2 \beta(1-\lambda) \int_{0}^{1} v^{\alpha-2} \log (1+v) d v
\end{aligned}
$$




$$
\begin{aligned}
& =1-2 \beta(1-\lambda)\left[\left.\log (1+v) \frac{v^{\alpha-1}}{\alpha-1}\right|_{0} ^{1}-\frac{1}{\alpha-1} \int_{0}^{1} \frac{v^{\alpha-1}}{1+v} d v\right] \\
& =1-2 \beta(1-\lambda)\left[\frac{\log 2}{\alpha-1}-\frac{F(1, \alpha ; \alpha+1 ;-1)}{\alpha(\alpha-1)}\right] \\
& \geq 1-2 \beta(\alpha)(1-\lambda)\left[\frac{\alpha \log 2-F(1, \alpha ; \alpha+1 ;-1)}{\alpha(\alpha-1)}\right]=\lambda .
\end{aligned}
$$

The desired conclusion follows.

THEOREM 2.6. Let $f \in \mathcal{A}$ satisfy the condition

$$
\operatorname{Re} z f^{\prime \prime}(z)>-\beta(1-\lambda), \quad 0<\beta \leq \frac{1}{2(2 \log 2-1)} \approx 1.29435
$$

Then $f \in \mathcal{P}(\lambda)$. In particular,

$$
\begin{aligned}
& \operatorname{Re} z f^{\prime \prime}(z)>-\beta \Rightarrow \operatorname{Re}\left(\frac{f(z)}{z}\right)>\frac{1-\log 2}{\log 2}=0.4427 \ldots \\
& \qquad \text { for } 0<\beta \leq 1 / \log 4 .
\end{aligned}
$$

Proof. Define $p(z)=f(z) / z$. Then $z^{2} p^{\prime \prime}(z)+2 z p^{\prime}(z)=z f^{\prime \prime}(z)$ and therefore, the desired conclusion follows from Lemma 2.5 , since $F(1,2 ; 3 ;-1)=$ $2(1-\log 2)$.

REMARK. From [1], we recall that if $\operatorname{Re} z f^{\prime \prime}(z)>-\beta$ for $0<\beta \leq$ $1 / \log 4 \approx 0.721348$, then $f \in \mathcal{S}^{*}$. We observe that $\mathcal{S}^{*}(1 / 2) \subsetneq \mathcal{P}(1 / 2)$. From Theorem 2.6, it follows that if $f \in \mathcal{A}$ satisfies the differential inequality

$$
\operatorname{Re}\left(z^{2} f^{\prime \prime \prime}(z)+2 z f^{\prime \prime}(z)\right)>-\beta
$$

then $\operatorname{Re} f^{\prime}(z)>0$ whenever $0<\beta \leq 1 /[4 \log 2-2]=\beta_{0} \approx 1.29435$. It is interesting to recall that if $f \in \mathcal{A}$ satisfies (2.12) then $f$ is convex whenever

$$
0<\beta \leq \beta_{\mathrm{c}}=1 / \log 4 \text {. }
$$

Note that $\beta_{0}>\beta_{\mathrm{c}}$ and we know that a convex function $f \in \mathcal{A}$ does not necessarily satisfy $\operatorname{Re} f^{\prime}(z)>0$ for $z \in \Delta$, and conversely, a function $f$ satisfying the last condition does not always have the convexity property. Indeed, even the assumption that $\left|f^{\prime}(z)-1\right|<\lambda$ in $\Delta$ does not necessarily imply that $f$ is starlike unless $\lambda \leq 2 / \sqrt{5}$ (see $[3,9]$ ).

Our next result, which is of independent interest, is a reformulated version of a result from [6] in our setting.

Theorem 2.7. Let $f \in \mathcal{A}_{n}=\left\{f \in \mathcal{A}: f(z)=z+a_{n+1} z^{n+1}+\cdots\right\}$ satisfy the condition

$$
\left|f^{\prime}(z)\left(\frac{z}{f(z)}\right)^{\mu+1}-1\right|<\lambda \quad(\lambda>0)
$$


and let

$$
g(z)=\int_{0}^{z}\left(\frac{\zeta}{f(\zeta)}\right)^{\mu} d \zeta
$$

(i) For $0<\mu<n$,

$$
g \in \mathcal{R}\left(1-\frac{\lambda \mu}{n-\mu}\right) .
$$

In particular, $\operatorname{Re} g^{\prime}(z)>0$ whenever $0<\mu \leq n /(1+\lambda)$.

(ii) For $\mu=n$,

$$
g \in \mathcal{R}\left(1-\frac{n\left|f^{(n+1)}(0)\right|}{(n+1) !}-n \lambda\right)
$$

In particular,

$$
\operatorname{Re} g^{\prime}(z)>0 \quad \text { whenever } \quad 0<\lambda \leq \frac{1}{n}-\frac{\left|f^{(n+1)}(0)\right|}{(n+1) !}
$$

Proof. For $\mu \in(0, n)$ and $f(z) \neq 0$ in $0<|z|<1$, we see that $g^{\prime}(z)=$ $(z / f(z))^{\mu}$ and

$$
z g^{\prime \prime}(z)=\mu\left(\frac{z}{f(z)}\right)^{\mu-1}\left[-\left(\frac{z}{f(z)}\right)^{2} f^{\prime}(z)+\frac{z}{f(z)}\right]
$$

so that

$$
g^{\prime}(z)-\frac{1}{\mu} z g^{\prime \prime}(z)=\left(\frac{z}{f(z)}\right)^{\mu+1} f^{\prime}(z)
$$

By hypothesis, we can write

$$
g^{\prime}(z)-\frac{1}{\mu} z g^{\prime \prime}(z)=1+\lambda w(z)
$$

where $w \in \mathcal{B}_{n}$. Suppose that $g^{\prime}(z)=1+\sum_{k=n}^{\infty} p_{k} z^{k}$ and $w(z)=\sum_{k=n}^{\infty} b_{k} z^{k}$. Then

$$
g^{\prime}(z)-\frac{1}{\mu} z g^{\prime \prime}(z)=1+\sum_{k=n}^{\infty}\left(1-\frac{k}{\mu}\right) p_{k} z^{k} .
$$

A comparison of the coefficient of $z^{k}$ on both sides of (2.13) shows that

$$
\left(1-\frac{k}{\mu}\right) p_{k}=\lambda b_{k} \quad(k \geq n)
$$

so that

$$
g^{\prime}(z)=1+\lambda \sum_{k=n}^{\infty} \frac{b_{k}}{1-k / \mu} z^{k} .
$$

Since $0<\mu<n$, we can rewrite the last equality in integral form

$$
g^{\prime}(z)=1-\lambda \int_{1}^{\infty} w\left(t^{-1 / \mu} z\right) d t
$$


and therefore (using $|w(z)| \leq|z|^{n}$ for $z \in \Delta$ ), it follows that

$$
\left|g^{\prime}(z)-1\right|<\lambda \int_{1}^{\infty} t^{-n / \mu} d t=\frac{\lambda \mu}{n-\mu},
$$

which gives the required conclusion. In particular, for $0<\mu \leq n /(1+\lambda)$, we have $\operatorname{Re} g^{\prime}(z)>0$ for $z \in \Delta$.

For the case $\mu=n$, proceeding as above but with $w(z)=\sum_{k=n+1}^{\infty} b_{k} z^{k}$, we get the required result.

Theorem 2.8. Let $f \in \mathcal{A}, 0<\alpha \leq 1$, and $\lambda>(1-\alpha) \sin (\pi \alpha / 2)$. Suppose that $f^{\prime}(z) f(z) / z \neq 0$ on $\Delta$ and

$$
\left|\operatorname{Im}\left[\lambda \frac{z f^{\prime \prime}(z)}{f^{\prime}(z)}+(1-\lambda) \frac{z f^{\prime}(z)}{f(z)}\right]\right|<\beta(\alpha, \lambda)
$$

where

$$
\beta(\alpha, \lambda)=\frac{\lambda}{2}\left[(\alpha+1) \frac{1}{t_{0}}+(\alpha-1) t_{0}\right]
$$

and $t_{0}$ is the pointwise solution of the equation

$$
2 t^{1+\alpha} \sin (\alpha \pi / 2)-\lambda\left(1-t^{2}\right)=0 .
$$

Then $f \in \mathcal{S}_{\alpha}^{*}$.

Proof. Define

$$
\frac{z f^{\prime}(z)}{f(z)}=\left(\frac{1+w(z)}{1-w(z)}\right)^{\alpha}
$$

It suffices to prove that $|w(z)|<1$ for $z \in \Delta$. Logarithmic differentiation of (2.15) gives

$$
1+\frac{z f^{\prime \prime}(z)}{f^{\prime}(z)}=\left(\frac{1+w(z)}{1-w(z)}\right)^{\alpha}+\alpha \frac{2 z w^{\prime}(z)}{1-w^{2}(z)}
$$

and therefore,

(2.16) $\lambda\left(1+\frac{z f^{\prime \prime}(z)}{f^{\prime}(z)}\right)+(1-\lambda) \frac{z f^{\prime}(z)}{f(z)}=\left(\frac{1+w(z)}{1-w(z)}\right)^{\alpha}+\alpha \lambda \frac{2 z w^{\prime}(z)}{1-w^{2}(z)}$.

Suppose it is not true that $|w(z)|<1, z \in \Delta$. Then there exists a $z_{0} \in \Delta$ such that $\left|w\left(z_{0}\right)\right|=1$ and, by Jack's well known lemma, $z_{0} w^{\prime}\left(z_{0}\right)=k w\left(z_{0}\right)$ with $k \geq 1$. If we put $w\left(z_{0}\right)=e^{i \theta}$, then from (2.16), we obtain

$$
\begin{aligned}
\lambda\left(1+\frac{z_{0} f^{\prime \prime}\left(z_{0}\right)}{f^{\prime}\left(z_{0}\right)}\right)+(1-\lambda) \frac{z_{0} f^{\prime}\left(z_{0}\right)}{f\left(z_{0}\right)} & =\left(\frac{1+e^{i \theta}}{1-e^{i \theta}}\right)^{\alpha}+\alpha \lambda \frac{2 k e^{i \theta}}{1-e^{2 i \theta}} \\
& =(i \cot (\theta / 2))^{\alpha}+i \frac{\lambda k \alpha}{\sin \theta}
\end{aligned}
$$


We consider first the case $0<\theta<\pi$. Then taking the imaginary part on both sides of (2.17), we get

$$
\begin{aligned}
\operatorname{Im}\left(\lambda \frac{z_{0} f^{\prime \prime}\left(z_{0}\right)}{f^{\prime}\left(z_{0}\right)}+(1-\lambda) \frac{z_{0} f^{\prime}\left(z_{0}\right)}{f\left(z_{0}\right)}\right) & =\cot ^{\alpha}(\theta / 2) \sin (\alpha \pi / 2)+\frac{\alpha \lambda k}{\sin \theta} \\
& \geq \cot ^{\alpha}(\theta / 2) \sin (\alpha \pi / 2)+\frac{\alpha \lambda}{\sin \theta} \\
& =t^{\alpha} \sin (\alpha \pi / 2)+\frac{\alpha \lambda}{2}\left(t+\frac{1}{t}\right) \\
& =: g(t), \quad \text { where } t=\cot (\theta / 2)>0 .
\end{aligned}
$$

We have

$$
g^{\prime}(t)=\alpha t^{\alpha-1} \sin (\alpha \pi / 2)+\alpha \lambda / 2-\alpha \lambda /\left(2 t^{2}\right)
$$

and

$$
g^{\prime \prime}(t)=\alpha(\alpha-1) t^{\alpha-2} \sin (\alpha \pi / 2)+\alpha \lambda / t^{3}=\frac{\alpha}{t^{3}}\left[(\alpha-1) t^{1+\alpha} \sin (\alpha \pi / 2)+\lambda\right] .
$$

Since $\lim _{t \rightarrow 0+} g^{\prime}(t)=-\infty, g^{\prime}(1)=\alpha \sin (\alpha \pi / 2)>0$ and $g^{\prime \prime}(t)>0$ for $0<t \leq 1$ and $\lambda>(1-\alpha) \sin (\pi \alpha / 2)$, we conclude that the function $g(t)$ attains its minimum

$$
\beta(\alpha, \lambda)=g\left(t_{0}\right)=\frac{1}{2}\left[(\alpha+1) / t_{0}+(\alpha-1) t_{0}\right],
$$

where $t_{0} \in(0,1)$ is the smallest positive root of the equation $g^{\prime}(t)=0$, i.e.

$$
2 t^{1+\alpha} \sin (\alpha \pi / 2)+\lambda t^{2}-\lambda=0 .
$$

Thus

$$
\operatorname{Im}\left(\lambda \frac{z_{0} f^{\prime \prime}\left(z_{0}\right)}{f^{\prime}\left(z_{0}\right)}+(1-\lambda) \frac{z_{0} f^{\prime}\left(z_{0}\right)}{f\left(z_{0}\right)}\right) \geq \beta(\alpha, \lambda) .
$$

Similarly, for $-\pi<\theta<0$, we obtain

$$
\operatorname{Im}\left(\lambda \frac{z_{0} f^{\prime \prime}\left(z_{0}\right)}{f^{\prime}\left(z_{0}\right)}+(1-\lambda) \frac{z_{0} f^{\prime}\left(z_{0}\right)}{f\left(z_{0}\right)}\right) \leq-\beta(\alpha, \lambda) .
$$

A combination of these two inequalities shows that

$$
\left|\operatorname{Im}\left(\lambda \frac{z_{0} f^{\prime \prime}\left(z_{0}\right)}{f^{\prime}\left(z_{0}\right)}+(1-\lambda) \frac{z_{0} f^{\prime}\left(z_{0}\right)}{f\left(z_{0}\right)}\right)\right| \geq \beta(\alpha, \lambda),
$$

which contradicts the assumption of the theorem.

So, $|w(z)|<1$ for $z \in \Delta$, and from (2.15), this is equivalent to the assertion that $f \in \mathcal{S}_{\alpha}^{*}$.

For $\lambda=1$, we have

Corollary 2.9. Let $f \in \mathcal{A}$ be such that $f^{\prime}(z) f(z) / z \neq 0$ on $\Delta$ and

$$
\left|\operatorname{Im} \frac{z f^{\prime \prime}(z)}{f^{\prime}(z)}\right|<\beta(\alpha), \quad z \in \Delta,
$$


where $0<\alpha \leq 1$,

$$
\beta(\alpha)=\frac{1}{2}\left[(\alpha+1) \frac{1}{t_{0}}+(\alpha-1) t_{0}\right]
$$

and $t_{0}$ is the pointwise solution of the equation

$$
2 t^{1+\alpha} \sin (\alpha \pi / 2)-\left(1-t^{2}\right)=0 .
$$

Then $f \in \mathcal{S}_{\alpha}^{*}$.

EXAmple 2.1. For $\alpha=1$, we have the equation $(2+\lambda) t^{2}-\lambda=0$ with positive root $t_{0}=\sqrt{\lambda /(2+\lambda)}$ and $\beta(1, \lambda)=\sqrt{\lambda(2+\lambda)}$. Now, we have the following implication (see [4, p. 115]) for $f \in \mathcal{A}$ with $f^{\prime}(z) f(z) / z \neq 0$ on $\Delta$ :

$$
\left|\operatorname{Im}\left[\lambda \frac{z f^{\prime \prime}(z)}{f^{\prime}(z)}+(1-\lambda) \frac{z f^{\prime}(z)}{f(z)}\right]\right|<\sqrt{\lambda(2+\lambda)} \Rightarrow\left|\arg \left(\frac{z f^{\prime}(z)}{f(z)}\right)\right|<\frac{\pi}{2},
$$

i.e. $f \in \mathcal{S}^{*}$.

A simple computation shows that $\beta(\alpha, \lambda)$ in Theorem 2.8 is larger than $\alpha \lambda$, and $\beta(\alpha, \lambda)$ is independent of the root $t_{0}$ of the appropriate equation. Namely, if we let

then

$$
\phi(t):=\beta(\alpha, \lambda)=\frac{\lambda}{2}[(\alpha+1) / t+(\alpha-1) t]
$$

$$
\phi^{\prime}\left(t_{0}\right)=\frac{\lambda}{2 t_{0}^{2}}\left[-(\alpha+1)+(\alpha-1) t_{0}^{2}\right]=\frac{1}{2 t_{0}^{2}}\left[\left(t_{0}^{2}-1\right) \alpha-\left(1+t_{0}^{2}\right)\right]<0,
$$

since $0<t_{0}<1,0<\alpha \leq 1$ and $\lambda>0$. It means that $\phi(t)$ is a decreasing function of $t_{0} \in[0,1]$ and we have

$$
\phi\left(t_{0}\right)>\phi(1)=\alpha \lambda
$$

\section{References}

[1] R. M. Ali, S. Ponnusamy and V. Singh, Starlikeness of functions satisfying a differential inequality, Ann. Polon. Math. 61 (1995), 135-140.

[2] P. N. Chichra and R. Singh, Complex sum of univalent functions, J. Austral. Math. Soc. 14 (1972), 503-507.

[3] R. Fournier, On integrals of bounded analytic functions in the closed unit disc, Complex Var. Theory Appl. 11 (1989), 125-133.

[4] S. Ponnusamy, Some applications of differential subordination and convolution techniques to univalent functions theory, Ph.D. thesis, I.I.T. Kanpur, 1988.

[5] S. Ponnusamy and S. Sabapathy, Polylogarithms in the theory of univalent functions, Results Math. 30 (1996), 136-150.

[6] S. Ponnusamy and P. Sahoo, Geometric properties of certain linear integral transforms, Bull. Belg. Math. Soc. Simon Stevin 12 (2005), 95-108.

[7] S. Ponnusamy and V. Singh, Criteria for univalent, starlike and convex functions, ibid. 9 (2002), 511-531. (Also Preprint 265, 2001, Department of Mathematics, University of Helsinki.) 
[8] R. Singh and S. Paul, Linear sums of certain analytic functions, Proc. Amer. Math. Soc. 99 (1987), 719-725.

[9] V. Singh, Univalent functions with bounded derivative in the unit disc, Indian J. Pure Appl. Math. 8 (1977), 1370-1377.

[10] - On some problems of Mocanu type, Indian J. Pure Appl. Math. 32) (2001), $1859-1867$.

[11] S. Y. Trimble, The convex sums of convex functions, Math Z. 109 (1969), 112-114.

[12] K.-J.Wirths, Bemerkungen zu einem Satz von Fejér, Anal. Math. 1 (1975), 313-318.

Department of Mathematics

Faculty of Technology and Metallurgy

4 Karnegijeva St.

11000 Belgrad

Serbia and Montenegro

E-mail: obrad@elab.tmf.bg.ac.yu
Department of Mathematics Indian Institute of Technology Madras Chennai-600 036, India E-mail: samy@iitm.ac.in vasu2kk@yahoo.com

Reçu par la Rédaction le 17.10.2003

Révisé le 30.6.2005 